\title{
Platelet associated immunoglobulins in primary biliary cirrhosis: a cause of thrombocytopenia?
}

\author{
M F BASSENDINE, J D COLLINS, J STEPHENSON, \\ P SAUNDERS, AND O F W JAMES \\ From the Department of Medicine, University of Newcastle upon Tyne and Department of Haematology, \\ General Hospital, Newcastle upon Tyne
}

SUMmARY Thrombocytopenia in cirrhotic patients is usually attributed to splenic pooling whereas in idiopathic thrombocytopenic purpura it is related to platelet bound immunoglobulin (PA-IgG). Since primary biliary cirrhosis (PBC) is an autoimmune disorder we have undertaken a prospective study to assess the frequency and possible relationship of PA-IgG to thrombocytopenia in this condition. Sixty two primary biliary cirrhosis patients ( 28 precirrhotic; 34 cirrhotic) were studied. Twenty five patients $(40 \%)$ had raised PA-IgG of whom 18 had cirrhosis. There was a significant inverse correlation between platelet count and PA-IgG $(\mathrm{p}<0.001)$ and between platelet count and spleen size $(\mathrm{p}<0.001)$. Thrombocytopenia (platelets $\left.<100 \times 10^{9} / 1\right)$ was found in nine patients $(15 \%)$; all nine had raised PA-IgG and eight were cirrhotic with an enlarged spleen. Two cirrhotic patients with persistent thrombocytopenia and bleeding episodes were treated with prednisolone and showed a useful therapeutic response. These results suggest that immune mediated platelet destruction and splenic pooling of platelets may both play a part in the thrombocytopenia observed in primary biliary cirrhosis.

Thrombocytopenia in patients with primary biliary cirrhosis has mainly been attributed to splenic pooling in an enlarged spleen, secondary to portal hypertension, ${ }^{1}$ although other factors, such as intrinsic platelet abnormalities leading to reduced survival have also been implicated. ${ }^{2}$ On the other hand thrombocytopenia in patients with idiopathic thrombocytopenic purpura is now recognised as being autoimmune in nature; there is strong evidence that an IgG autoantibody directed against platelet antigens causes increased platelet destruction. ${ }^{3-5}$ Progress in this field has been hampered by the difficulty of studying the binding of antibody to platelets but in recent years several techniques which measure platelet associated immunoglobulin (PA-IgG) have been developed. ${ }^{6-9}$ The more widespread application of this investigation has resulted in the demonstration of PA-IgG in other conditions such as systemic lupus erythematosis,${ }^{10}$ neoplastic disorders ${ }^{11}$ chronic active hepatitis ${ }^{12}$ and alcoholic cirrhosis $;{ }^{13}$ these findings add to the suggestion that immune mechanisms may mediate thrombocyto-

Address for correspondence: Dr O F W James, Medical Unit 1, Freeman Hospital, Newcastle upon Tyne NE7 7DN.

Received for publication 21 November 1984 penia in many more disorders than was previously thought likely.

Primary biliary cirrhosis ('chronic nonsuppurative destructive cholangitis') is an autoimmune disease characterised by many immunological abnormalities including the presence of autoantibodies in the serum ${ }^{14}$ and hypergammaglobinaemia, ${ }^{15}$ and associated with other autoimmune disorders such as thyroid disease. ${ }^{16}$ Raised PA-IgG has been reported in one patient with primary biliary cirrhosis who had thrombocytopenia, ${ }^{13}$ suggesting that immune thrombocytopenia may also develop in this condition. In view of the potential therapeutic implications of this finding, we have carried out a prospective study to assess the frequency of PA-IgG in primary biliary cirrhosis and its possible relationship to thrombocytopenia in this condition.

\section{Methods}

PATIENTS

Sixty two patients (57 women, five men) with primary biliary cirrhosis were studied; the diagnosis being established on standard biochemical, immunological and histological criteria. ${ }^{16}$ Thirty four 
patients were cirrhotic and 28 precirrhotic on liver biopsy. An estimate of splenomegaly was made (grade $0-3$ ) by an independent observer (JDC) on a standard ${ }^{99}$ technetium liver and spleen scan. Two cirrhotic primary biliary cirrhosis patients with persistent thrombocytopenia, epistaxis and gastrointestinal bleeding in whom an immune mediated destruction of platelets was suspected were treated with prednisolone therapy and their clinical course carefully monitored.

Full blood counts and platelet counts in all patients were carried out on a Coulter Counter, Model S plus III. Platelet associated immunoglobulins were estimated using a modification of a method previously described using ${ }^{125}$ I-labelled Staphylococcal Protein A (SPA). ${ }^{17-19}$

Platelets from patients with primary biliary cirrhosis were harvested by differential centrifugation, washed in phosphate buffered saline pH $7 \cdot 2$ (PBS) and stored at $4^{\circ} \mathrm{C}$ in phosphate buffered saline containing $15.4 \mathrm{mmol} / \mathrm{l}$ sodium azide. Normal platelets from at least 10 group $\mathrm{O}$ blood donors were similarly harvested and pooled ('pooled normal platelets'). Staphylococcal Protein A (Pharmacia Ltd - Uppsala) was radio-iodinated by the chloramine $\mathrm{T}$ method with $\mathrm{Na}^{125} \mathrm{I}$ (Amersham International) and stored in $20 \mu \mathrm{l}$ aliquots at $-40^{\circ} \mathrm{C}$. This stock radiolabelled SPA was diluted appropriately before use. Duplicate aliquots of either test or pooled normal platelets containing $10^{8}$ platelets were resuspended in and incubated with $100 \mu \mathrm{l}$ of working dilution of SPA ${ }^{125} \mathrm{I}$ at $4^{\circ} \mathrm{C}$ for 60 minutes. Unbound SPA ${ }^{125}$ I was removed by washing $\times 3$ in phosphate buffered saline. The platelet button was resuspended in phosphate buffered saline and bound radioactivity counted. The PA-IgG was reported as the ratio of $\operatorname{SPA}^{125} I$ bound to test platelets, compared with that bound to 'pooled normal platelets'. The normal range of PA-IgG for a cohort of 50 normal people was up to 1.65 (mean $0 \cdot 89 \pm 2 \mathrm{SD}(\mathrm{SD}=0 \cdot 38)$ ).

The Spearman rank correlation coefficient was used to test the correlation of platelet count with PA-IgG and with spleen size. The $\chi^{2}$ test was applied to analyse the association of thrombocytopenia (platelet count $<100 \times 10^{9} / 1$; normal range $\left.150-400 \times 10^{9} / 1\right)$ with raised PA-IgG ratio and with an enlarged spleen.

\section{Results}

The PA-IgG results in all patients tested are shown in Fig. 1. There were raised levels of PA-IgG in 25 patients with primary biliary cirrhosis $(40 \%)$, the range of values was 0.8 to 16 (mean 1.8 ). Raised PA-IgG was more commonly found in cirrhotic than

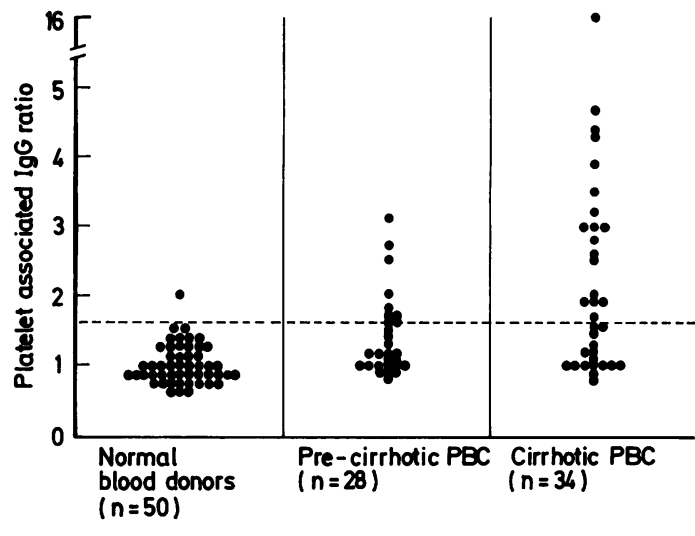

Fig. 1 Platelet-associated IgG ratio in patients with primary biliary cirrhosis and normal controls. Line represents upper limit of normal range (mean $\pm 2 S . D$.).

pre-cirrhotic patients (53\% vs $25 \%$ ).

In the 62 primary biliary cirrhosis patients there was a significant inverse correlation between platelet count and PA-IgG ratio $(r=-0.51 \mathrm{p}<0.001)$ (Fig. 2) and between platelet count and spleen size $(r=-0.63 p<0.001)$. Nine primary biliary cirrhosis patients (eight with cirrhosis) had platelet counts below $100 \times 10^{9} / 1$ for at least three months. This thrombocytopenia was significantly associated with both raised PA-IgG $(9 / 9, \mathrm{p}<0.0005)$ and an enlarged spleen $(8 / 9, p<0 \cdot 005)$. (Figs $3 a$ and $b)$.

The clinical course of the two primary biliary cirrhosis patients with persistent thrombocytopenia, epistaxis and gastrointestinal bleeding who were treated with prednisolone is shown in Fig. 4. Patient

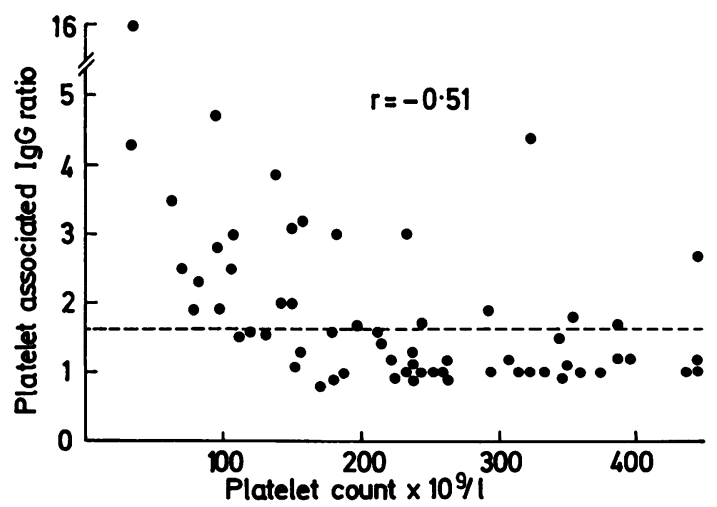

Fig. 2 Relationship between PA-IgG and platelet count in primary biliary cirrhosis patients. Line represents upper limit of normal range (mean \pm 2 S.D.). 

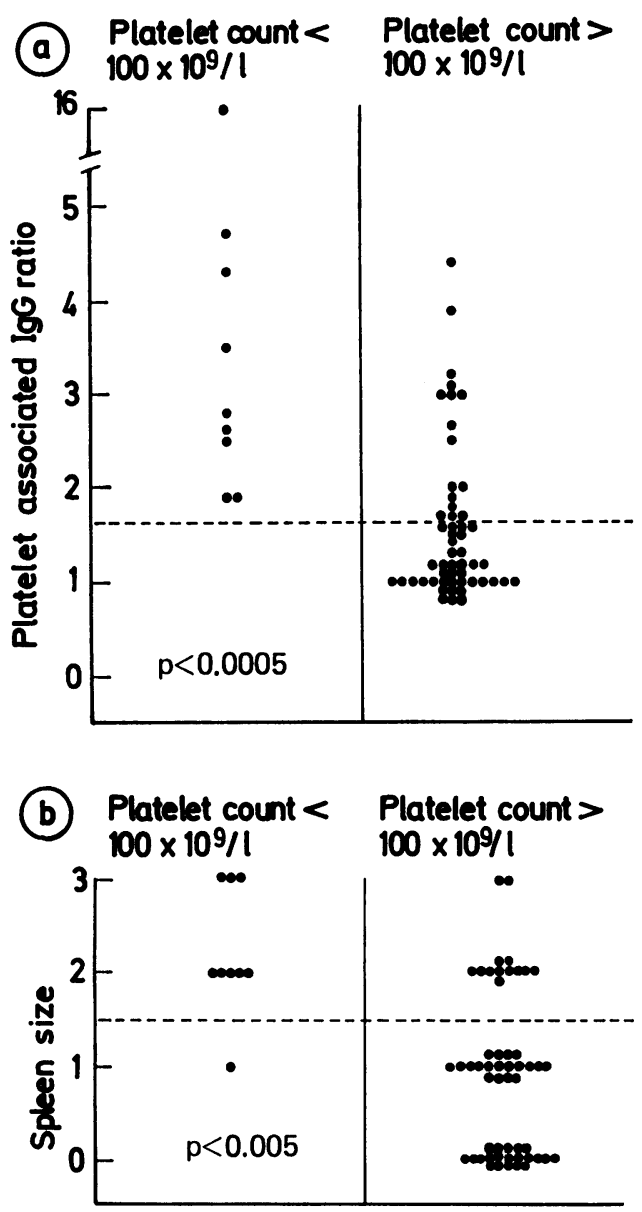

Fig. 3 (A) Relationship between PA-IgG and thrombocytopenia (platelets $<100 \times 10^{\circ} / \mathrm{l}$ ) in primary biliary cirrhosis patients. Line represents upper limit of normal range (mean $\pm 2 S$. D.).

(b) Relationship between spleen size and thrombocytopenia (platelets $<100 \times 10^{9} / \mathrm{ll}$ ) in primary biliary cirrhosis patients. Line represents division between considerable splenomegaly (spleen size 2 \& 3) normal or slight splenomegaly (spleen size 0 \& 1).

1 (Fig. 4a) showed a dramatic therapeutic response with a marked improvement in her platelet count and cessation of bleeding. When her immunosuppressive therapy was stopped her platelet count slowly fell towards pretreatment levels, associated with a raised PA-IgG of $4 \cdot 3$. Patient 2 (Fig. 4b) followed a similar though less remarkable clinical course. When prednisolone was stopped her platelet count also fell, associated with a raised PA-IgG of 16 and epistaxis recurred. Restarting prednisolone therapy resulted in a partial clinical response with an increase in her platelet count associated with a decrease in PA-IgG to $2 \cdot 8$. No reactive thrombocytosis had been observed in either patient following previous bleeding episodes.

\section{Discussion}

The clinical value of PA-IgG estimations has been shown for a variety of disorders. A large number of radioisotopic methods have been devised for the quantitation of PA-IgG. The majority rely on a labelled anti-human IgG antibody for its detection. We have used ${ }^{125}$ I labelled staphylococcal protein A. This detects mainly IgG 1,2 and 4, although IgG 3 and IgM are detected to some extent. ${ }^{20}$ Evidence suggests that the predominant subclass of IgG found on platelets in patients with immune thrombocytopenia is IgG $1 .^{21} 22$ One recent study though has suggested a hitherto unrecognised frequent involvement of PA-IgM in ITP ${ }^{23}$ so it is possible the method used may underestimate total platelet-associated immunoglobulin.

Our study has shown that raised PA-IgG occurs in some patients with primary biliary cirrhosis and that they are more commonly associated with cirrhotic than non-cirrhotic patients. The pathogenic role of this platelet bound immunoglobulin is still far from clear. In ITP some studies have shown an inverse correlation between PA-IgG and platelet count ${ }^{6} 79$ but another study has been unable to confirm this. ${ }^{23}$ Similarly a significant inverse correlation between PA-IgG and platelet survival has been shown in idiopathic thrombocytopenic purpura, ${ }^{5}$ but has not been found in other thrombocytopenic patients. ${ }^{24}$ The application of PA-IgG measurements to these other patient groups has resulted in modification of the original assumption that raised PA-IgG levels indicate specific antiplatelet antibody. ${ }^{7}{ }^{7}$ It is now suggested that possibly only $10 \%$ of PA-IgG is directly related to a shortened platelet life $\operatorname{span}^{25}$ and that the remaining $60-90 \%$ of PA-IgG is either non-specifically associated with platelets or is directed towards easily solubilised platelet antigens. ${ }^{26}$ Some of the immunoglobulin bound to platelets in primary biliary cirrhosis could conceivably be related to changes in receptor availability and function, ${ }^{27}$ secondary to the altered lipid composition of platelets that occurs in chronic liver disease. ${ }^{28}$ Despite the immunological nature of primary biliary cirrhosis the incidence of PA-IgG in the cirrhotic patients $(53 \%)$ is lower than that previously reported in alcoholic cirrhosis. ${ }^{13}$ This is consistent with the concept that some of the PA-IgG detected in primary biliary cirrhosis is formed by a mechanism common to other forms of chronic liver 
(a)
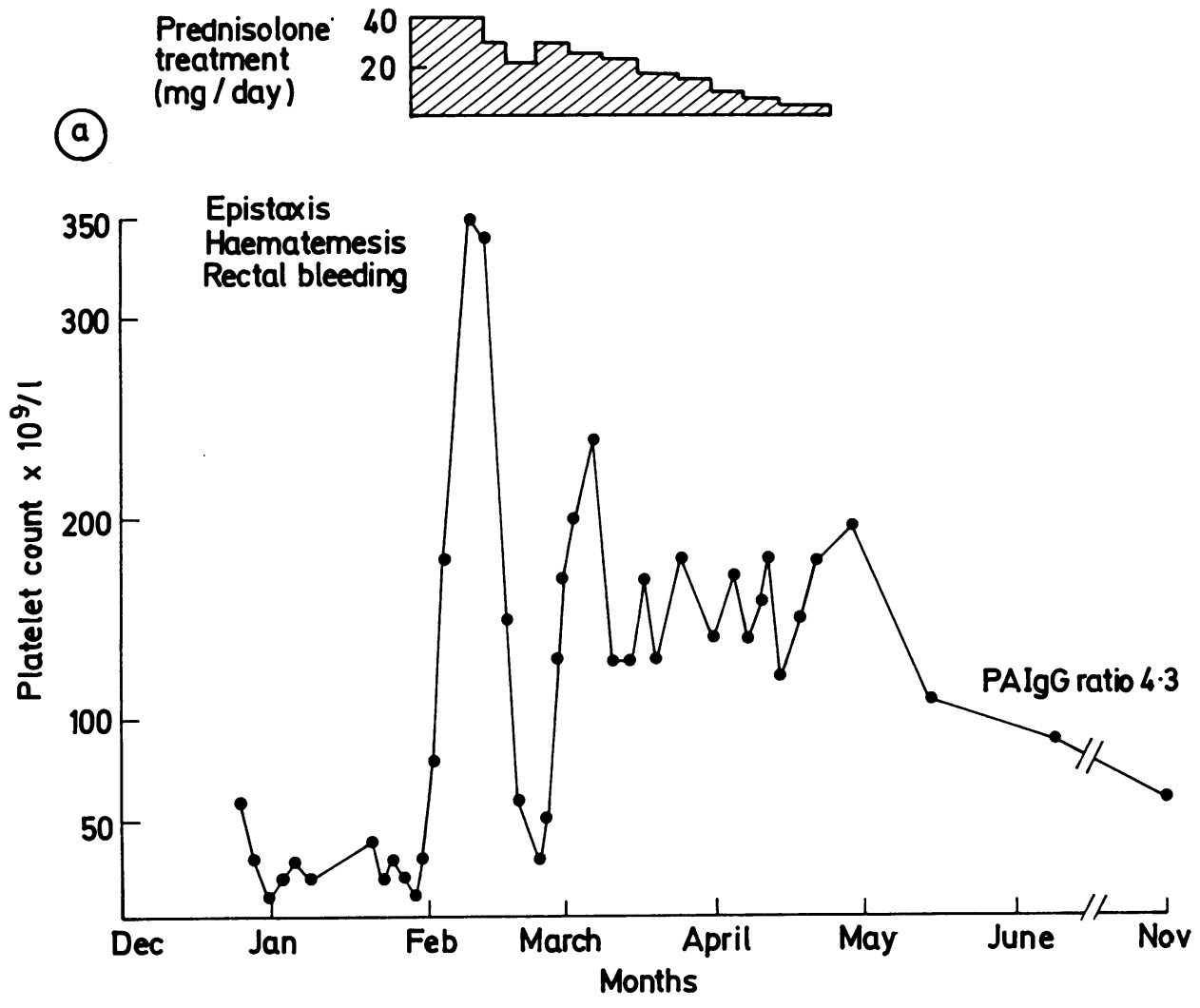

Prednisolone treatment ( $\mathrm{mg} /$ day)

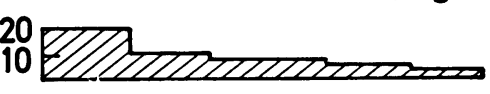

(b)
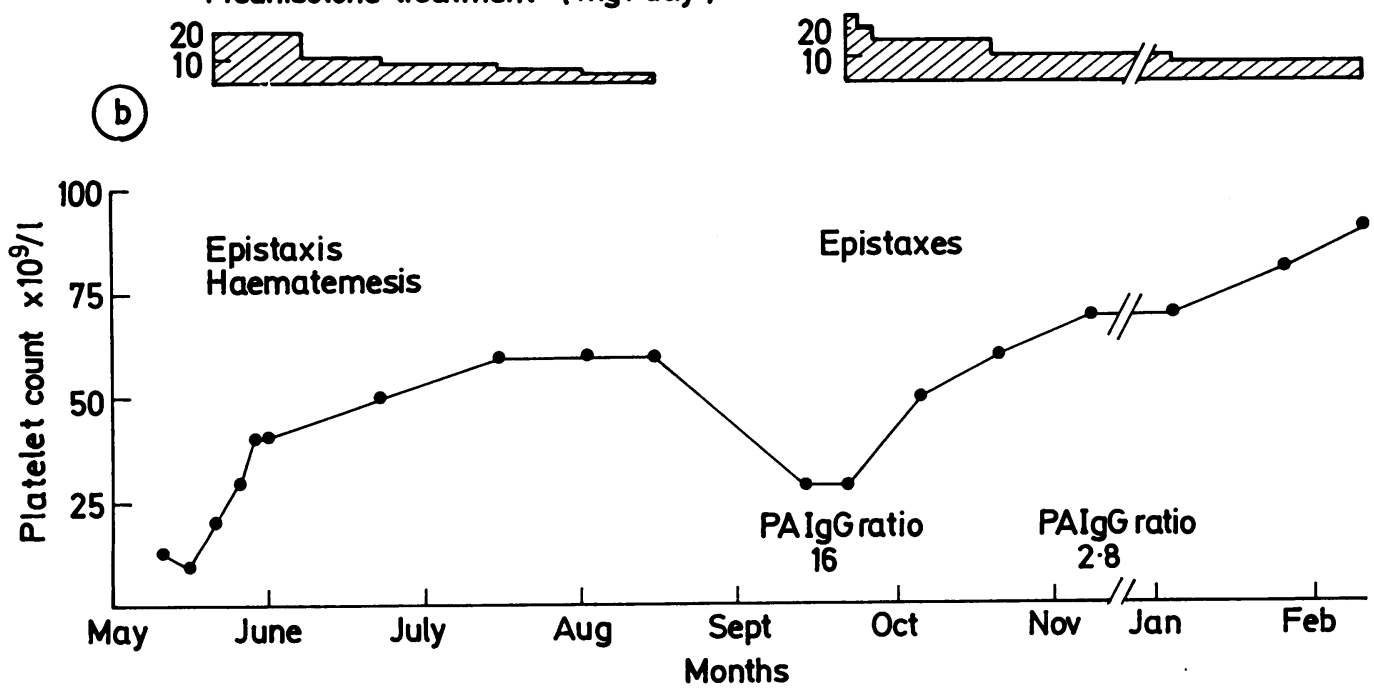

Fig. 4 Response of platelet count to steroid treatment in two primary biliary cirrhosis patients. 
disease, and may not be specifically 'autoimmune' in aetiology.

Nonetheless studies in other forms of chronic liver disease have not shown the inverse correlation between platelet count and PA-IgG, ${ }^{12}{ }^{13}$ which has been found in this study. This inverse correlation is similar to idiopathic thrombocytopenic purpura ${ }^{679}$ and suggests that immune mediated platelet destruction is contributing to the thrombocytopenia found in some primary biliary cirrhosis patients. Our finding of raised PA- IgG in all nine primary biliary cirrhosis patients with persistent thrombocytopenia also supports this hypothesis, and suggests that an immune mechanism may be as important a factor in thrombocytopenia as splenic pooling in an enlarged spleen. The possible immunological nature of platelet destruction is further supported by the two patients reported here who exhibited a useful therapeutic response to steroids. The increase in platelet count during immunosuppressive treatment and the raised PA-IgG when taken off therapy are both typical of classical idiopathic thrombocytopenic purpura, and suggest an immune pathogenesis for thrombocytopenia common to both diseases.

The significant inverse correlation of platelet count with spleen size also found in this study is consistent with previous data indicating that pooling of platelets in an enlarged spleen is a cause of thrombocytopenia. ${ }^{1}$ Our study, however, would suggest that this is an over simplication of the mechanisms involved and that immune mediated platelet destruction in the spleen plays an additive role.

A better understanding of the complex pathophysiology of thrombocytopenia in primary biliary cirrhosis and other forms of chronic liver disease may be reached by measurement of PA-IgG in conjunction with studies of platelet survival and sites of destruction. The finding of raised PA-IgG in thrombocytopenic patients can both raise the suspicion that immune mediated platelet destruction is occurring and suggest that successful management may include measures directed towards modification of the immune process.

\section{References}

1 Aster RH. Pooling of platelets in the spleen, role in the pathogenesis of hypersplenic thrombocytopenia. J Clin Invest 1966; 45: 645-57.

2 Rubin MH, Weston MJ, Langley PG, White Y, Williams R. Platelet function in chronic liver disease. Dig Dis Sci 1979; 24: 197-202.

3 Shulman NR, Marder VJ, Hiller MC, Collier EM. Platelet and leucocyte isoantigens and their antibodies: serologic, physiologic and clinical studies. In: Moor CV, Brown EB, eds. Progress in haematology. New York: Grune and Stratton, 1964; 222-304.

4 McMillan R. Chronic idiopathic thrombocytopenic purpura. N Engl J Med 1981; 304: 1135-47.

5 Kernoff LM, Blake KCH, Shackleton D. Influence of the amount of platelet bound IgG on platelet survival and site of sequestration in autoimmune thrombocytopenia. Blood, 1980; 55: 730-3.

6 Dixon R, Rosse W, Ebbert L. Quantitative determination of antibody in idiopathic thrombocytopenic purpura: correlation of serum and platelet bound antibody with clinical response. $N$ Engl J Med 1975; 292: 230-6.

7 Luiken GA, McMillan R, Lightsey AC et al. Platelet associated IgG in immune thrombocytopenic purpura. Blood 1977; 50: 317-25.

8 Cines DB, Schreiber AD. Immune thrombocytopenia. Use of a Coombs antiglobulin test to detect IgG and C3 in platelets. $N$ Engl J Med 1979; 300: 106-11.

9 Hymes K, Shulman S, Karpatkin S. A solid phase radioimmunoassay for bound antiplatelet antibody. Studies on 45 patients with autoimmune platelet disorders. J Lab Clin Med 1979; 94: 639-48.

10 Kelton JG, Powers PJ, Carter CJ. A prospective study of the usefulness of the measurement of plateletassociated IgG for the diagnosis of idiopathic thrombocytopenic purpura. Blood 1982; 60: 1050-3.

11 Schwartz KA, Slichter SJ, Harker LA. Immunemediated platelet destruction and thrombocytopenia in patients with solid tumours. Br J Haematol 1982; 51: 17-24.

12 Landolfi R, Leone G, Tedeli G et al. Platelet associated IgG in acute and chronic hepatic diseases. Scand $J$ Haematol 1980; 25: 417-22.

13 Barison IG, Knight ID, Viola L, Boots MA, MurrayLyon IM, Mitchell TR. Platelet associated immunoglobulins in chronic liver disease. Br J Haematol 1981; 48: $347-50$.

14 Doniach D, Roitt IM, Walker JG, Sherlock S. Tissue antibodies in primary biliary cirrhosis, active chronic hepatitis, cryptogenic cirrhosis and other liver disease and their implications. Clin Exp Immunol 1966; 1: 237-62.

15 MacSween RMM, Horne HW, Moffat AJ, Hughes HM. Serum protein levels in primary biliary cirrhosis. $J$ Clin Pathol 1972; 25: 789-94.

16 James OFW, Macklon AF, Watson AJ. Primary biliary cirrhosis - a revised clinical spectrum. Lancet 1981; 1: 1278-81.

17 Kekomaki R. Detection of platelet bound IgG with ${ }^{125}$ I-labelled Staphylococcal Protein A. Med Biol 1977; 54: $112-4$.

18 Bergh OJ, Solheim BG. Detection of thrombocyte antibodies by ${ }^{125} \mathrm{I}$-labelled protein A. Tissue Antigens 1978; 12: 189-94.

19 Shaw GM, Axelson J, Maglott JG, LoBuglio AF. Quantification of platelet-bound IgG by ${ }^{125} \mathrm{I}$ Staphylococcal protein A. In Immune thrombocytopenic purpura and other thrombocytopenic disorders. Blood 1984; 63: 154-61.

20 Lindmark R, Thoren-Tolling K, Sjoquist J. Binding of immunoglobulins to Protein $\mathrm{A}$ and immunoglobulin 
levels in mammalian sera. J Immunol Meth 1983; 61: 1-13.

21 Borne AEG von dem, Helmerholst FM, Leenwen EF von, Pegels HG, Riexz E von, Engafriet CP. Autoimmune thrombocytopenia: detection of platelet autoantibodies with the suspension immunofluorescence test. Br J Haematol 1980; 45: 319-27.

22 Rosse WF, Adams JP, Yonat WJ. Subclasses of IgG antibodies in immune thrombocytopenic purpura (ITP). Br J Haematol 1980; 46: 109-14.

23 Nel JD, Stevens K, Mouton A, Pretomis FJ. Plateletbound IgM in autoimmune thrombocytopenia. Blood 1983; 61: 119-24.

24 Mueller-Eckhardt C, Mueller-Eckhardt G, Kayser W, Voss RM, Wegner J, Kuenzlen E. Platelet associated $\mathrm{IgG}$, platelet survival and platelet sequestration in thrombocytopenic states. $\mathrm{Br} J$ Haematol 1982; 52: 49-58.

25 Karpatkin S, Siskind GW. Studies on the specificity of antiplatelet antibodies. Proc Soc Exp Biol Med 1974; 147: 715-9.

26 Pfueller SL. Cosgrove L, Firkin BG, Tew D. Relationship of raised platelet IgG in thrombocytopenia to total platelet protein content. Br J Haematol 1981; 49: 293-302.

27 Cooper RA. Abnormalities of cell-membrane fluidity in the pathogenesis of disease. N Engl J Med 1977; 297: 371-7.

28 Owen JS, Hetton RA, Day RC, Bruckdorfer R, McIntyre N. Platelet lipid composition and platelet aggregation in human liver disease. $J$ Lipid Res 1981; 22: $423-30$. 